\title{
Variabilidade espacial dos atributos químicos do solo, associada ao microrrelevo
}

\author{
Adriana G. Artur ${ }^{1}$, Daniel P. Oliveira ${ }^{2}$, Mirian C. G. Costa ${ }^{3}$, Ricardo E. Romero ${ }^{4}$, \\ Marcus V. C. Silva ${ }^{5} \&$ Tiago O. Ferreira ${ }^{6}$
}

${ }^{1}$ UFC. Fortaleza, CE. E-mail: driguirado@yahoo.com.br. Bolsista DCR (Autor correspondente)

${ }^{2}$ UFC. Fortaleza, CE. E-mail: daniel_pontes78@hotmail.com

${ }^{3}$ UFC. Fortaleza, CE. E-mail: mirian.costa@ufc.br

${ }^{4}$ UFC. Fortaleza, CE. E-mail: reromero@ufc.br

${ }^{5}$ UECE. Fortaleza, CE. E-mail: mvcs81@hotmail.com

${ }^{6}$ ESALQ/ USP. Piracicaba, SP. E-mail: toferreira@usp.br

\section{Palavras-chave:}

fertilidade do solo

fluxo de água

vegetação

\begin{abstract}
R E S U M O
A variabilidade dos atributos químicos do solo é influenciada por complexas interações entre os fatores e os processos de formação do solo. Este trabalho partiu da hipótese de que os atributos químicos do solo variam com o microrrelevo a ponto de alterar as recomendações de manejo para uso agrícola. Com isto, o relevo foi separado em compartimentos e as superfícies estudadas com o objetivo de se determinar os atributos químicos do solo, avaliar a distribuição e a dependência espacial desses atributos. A grade amostral compreendeu uma área de 102 ha da Fazenda Agrícola Famosa, localizada na Chapada do Apodi, CE. O solo da área apresenta origem calcária e as amostras foram coletadas em 92 pontos distanciados $200 \mathrm{~m}$ na linha e $100 \mathrm{~m}$ entre linhas, nas profundidades de 0-20 e 20-40 cm. Com os dados, gerou-se um modelo de elevação do terreno e, com base nas formas de relevo identificadas, dividiu-se o local de estudo em superfícies. Os dados foram avaliados por estatística descritiva e análise de variabilidade espacial, com base no ajuste de semivariogramas e mapas de krigagem. Os atributos químicos do solo apresentaram variabilidade espacial nos microrrelevos convexo, retilíneo e côncavo, intensificada pelo fluxo de água nas profundidades.
\end{abstract}

Key words: soil fertility water flow crop

\section{Spatial variability of soil chemical attributes associated with microrelief}

\begin{abstract}
A B S T R A C T
The variability of soil chemical attributes is influenced by complex interactions between factors and processes of soil formation. This study hypothesized that soil chemical attribute varies with microrelief to change management recommendations for agricultural use. The relief was separated in compartments and surfaces were studied with the objective to determine the soil chemical properties and to evaluate the spatial dependence of these attributes. Sampling grid was established in an area of 102 ha at Agricultural Farm Famosa localized in Plateau of Apodi, CE. The soil was derived from limestone and was collected at 92 points spaced at 200 by $100 \mathrm{~m}$ and in $0-20$ and 20-40 $\mathrm{cm}$ depths. With the topographic data a terrain modeling was generated and based on the identified microrelief, the study site was divided into surfaces. Data were analysed using descriptive statistics and spatial variability analysis, with the semivariograms adjustments and kriging maps. Soil chemical attributes showed spatial variability at the convex, concave and rectilinear microrelief, being intensified by water flow at the depths.
\end{abstract}

\section{INTRODUÇÃO}

Solos sob vegetação natural apresentam variabilidade em seus atributos químicos (Montezano et al., 2006), resultante dos processos de formação e esta variabilidade varia tanto no sentido horizontal como no vertical. Além da variabilidade natural do solo, as práticas agrícolas de manejo e uso das terras são fatores adicionais de variabilidade. Estudos já foram realizados enfatizando variações causadas pelo próprio uso e manejo (Mello et al., 2006; Zanão Júnior et al., 2010), mas são escassas as informações que tratam desse tipo de variabilidade associada ao microrrelevo. Para Marques Júnior \& Lepsch (2000), pequenas variações no gradiente do declive já são suficientes para causar variabilidade.

De acordo com Daniels \& Hammer (1992), o fluxo, a distribuição sazonal e a quantidade de água são processos causadores de variabilidade espacial de atributos do solo pois podem condicionar ambientes com características diferenciadas, a partir da interferência no movimento de bases trocáveis.

O relevo é o fator de formação do solo responsável por alterar sua umidade, a intensidade de ocorrência dos processos de 
erosão e lixiviação, seu microclima e o nível do lençol freático. A posição que o solo ocupa na paisagem tem influência na variabilidade dos atributos (Franzen et al., 2006).

A declividade e a pedoforma afetam os seguintes parâmetros do solo: fluxo e transporte de água (Souza et al., 2006), absorção e capacidade de armazenamento de água, diversidade de espécies animais e vegetais, redistribuição (erosão e /ou deposição) das partículas do solo, teores de matéria orgânica (MO) e nutrientes resultando em alterações nos atributos do solo de maneira a afetar as possibilidades de uso da terra, influenciando a eficiência das práticas de manejo, tal como o desenvolvimento das culturas.

As superfícies que compõem o microrrelevo (convexas, retilíneas e côncavas) exercem influência na exposição do material de origem, na intensidade e no sentido do fluxo da água no perfil do solo. Isto altera atributos químicos, físicos e biológicos e esta alteração pode ser intensificada com práticas de manejo do solo (Guo et al., 2007). Assim, o estudo dessas superfícies torna-se premissa básica nos levantamentos pedológicos, na tomada de decisões em relação ao manejo do solo, intensidade e frequência de amostragem e gerenciamento de práticas agrícolas e avaliação dos efeitos da agricultura sobre a qualidade ambiental (Corá et al., 2004).

O presente estudo foi realizado para comprovar a hipótese de que os atributos químicos do solo variam com o microrrelevo, a ponto de alterar as recomendações de manejo para uso agrícola. Com isto, o relevo foi separado em compartimentos e as pedoformas foram estudadas com o objetivo de se determinar os atributos químicos do solo, avaliar a distribuição e a dependência espacial desses atributos da região da Chapada do Apodi - CE, com o intuito de fornecer subsídios para o manejo adequado da fertilidade do solo com vista à cultura a ser estabelecida.

\section{MATERial e Métodos}

O estudo foi realizado em uma área de 102 ha da Fazenda Agrícola Famosa, localizada na Chapada do Apodi (Limoeiro do Norte, CE). O clima da região, segundo a classificação de Köppen, é do tipo BSw'h', tropical quente semiárido, com pluviosidade média anual de 550 a $940 \mathrm{~mm}$ e temperatura média anual de $23{ }^{\circ} \mathrm{C}$, marcado por dois períodos bem definidos: seco (prolongado) e úmido (curto e irregular). A vegetação local é caracterizada como caatinga hiperxerófila.

A Chapada do Apodi apresenta relevo plano e homogêneo e está inserida na Bacia do Rio Potiguar, com ocorrência de calcário da Formação Jandaíra no topo e arenito Açu na base (Brasil, 1973). Isto influencia na mineralogia dos solos da região os quais são classificados como eutróficos, podendo apresentar textura argilosa ou franco-argilosa, além de boa fertilidade natural.

O levantamento planialtimétrico para a geração de mapas de fertilidade foi feito utilizando-se o método DGPS (método do posicionamento diferencial - Differential Global Positioning System), em que a estação móvel (GPS) faz conexão com a estação de referência, eliminando erros. A grade estruturada para amostragem do solo na área de estudo foi a de malha de tamanho regular e tipo trapezoidal, com os pontos distanciados $200 \mathrm{~m}$ na linha e $100 \mathrm{~m}$ entre linhas, totalizando nove linhas (Figura 1). A partir dos dados altimétricos foi gerado um Modelo de Elevação Digital (MED) no software Surfer 8.0 e realizada a compartimentação do relevo considerando-se a curvatura das superfícies da área (Figura 1).

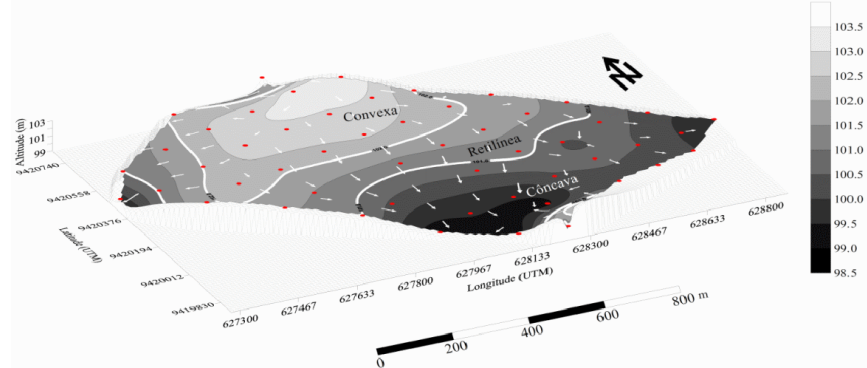

Figura 1. Compartimentação do microrrelevo e transporte superficial das águas (setas)

Em cada ponto da grade foram coletadas amostras de solo em duas profundidades (0-20 e $20-40 \mathrm{~cm})$, totalizando 92 amostras por profundidade, sendo 36 amostras de solo na superfície côncava; 29 na convexa e 27 na retilínea; após a coleta as amostras de solo foram secadas, passadas em peneiras de abertura de malha de $4 \mathrm{~mm}$, homogeneizadas e submetidas à análise para caracterização química. Foram feitas as seguintes determinações: $\mathrm{pH}$ em água; potássio $(\mathrm{K})$; cálcio $(\mathrm{Ca})$; magnésio $(\mathrm{Mg})$; sódio $(\mathrm{Na})$ e acidez potencial $(\mathrm{H}+\mathrm{Al})$, conforme os métodos descritos em por Claessen (1997). Por cálculo, foram determinadas a capacidade de troca catiônica (CTC) a pH 7,0 e a saturação por bases (V\%); a profundidade dos solos foi determinada por meio de tradagens.

Os dados obtidos em laboratório foram submetidos à análise exploratória para o cálculo de medidas de localização (média, mediana), de variabilidade (coeficiente de variação) e de tendência central (assimetria); em seguida verificou-se a normalidade dos atributos (Cruz et al., 2010).

Para avaliação da variabilidade espacial dos atributos químicos foram feitas análises variográficas cujos dados foram ajustados aos modelos esférico, gaussiano e exponencial (Oliveira et al., 2009). Após obtenção dos semivariogramas e constatação da dependência espacial, mapas de isolinhas foram gerados utilizando-se, como interpolador (preditor) o método da Krigagem por meio do programa Surfer 8.0 (Golden Software Inc., 1999).

A classificação do grau de dependência espacial (GDE) foi feita com base na razão entre o efeito pepita (Co) e o patamar $(\mathrm{Co}+\mathrm{C})$, sendo considerada fraca para GDE superior a $75 \%$, moderada entre 25 e $75 \%$ e forte para GDE inferior a $25 \%$ (Cambardella et al., 1994).

\section{Resultados e Discussão}

A partir do modelo de elevação digital foram estabelecidos três compartimentos na área de estudo: superfície convexa, 
retilínea e côncava. Na Figura 1 pode ser observado o sentido preferencial do fluxo de água na área, indicado pelas setas. No microrrelevo convexo parte da água é transportada para a superfície retilínea e, desta, para a côncava. O fluxo de água (convergência e divergência) está relacionado com a inclinação e curvatura da área, causando variações nos atributos do solo. Essas variações estão relacionadas ao fato de que a drenagem do solo está ligada aos ambientes deposicionais e erosionais direcionando, assim, o caminhamento da água na área (Nizeyimana \& Bicki, 1992).

O microrrelevo influencia na drenagem e no movimento horizontal e vertical da água no perfil, acelerando as reações químicas do intemperismo e promovendo o transporte de sólidos ou de materiais em solução. Assim, há aumento da variabilidade espacial dos atributos químicos do solo e favorecimento da identificação de superfícies interdependentes em relação ao processo pedogenético (Bockheim et al., 2005). Isto significa que o movimento da água na paisagem é o principal responsável pelo processo de desenvolvimento do solo (Gobin et al., 2001), por condicionar ambientes com características diferenciadas e por interferir no movimento de bases.

Os parâmetros referentes à análise descritiva dos dados para cada atributo químico avaliado estão apresentados nas Tabelas 1 e 2 . Os valores de média e mediana da maioria dos atributos químicos do solo nas profundidades de 0-20 e 20-40 $\mathrm{cm}$ estão próximos, evidenciando que estes apresentam ou se aproximam da distribuição normal, ou seja, medidas de tendência central não são dominadas por valores atípicos na distribuição (Cambardella et al., 1994) podendo ser aplicada a geoestatística.
A variabilidade dos atributos químicos pode ser classificada em função do seu coeficiente de variação (CV). Segundo a classificação de Warrick \& Nielsen (1980), na profundidade de 0-20 cm, independentemente do compartimento, os CVs foram baixos para os valores de $\mathrm{pH}$, altos para $\mathrm{Na}^{+}$e $\mathrm{H}+$ Al e médios para os demais atributos químicos (Tabela 1). Tendência semelhante foi observada na camada de $20-40 \mathrm{~cm}$, com exceção do $\mathrm{H}+\mathrm{Al}$ que teve coeficiente de variação médio para a superfície côncava (Tabela 2) devido à menor variação desta característica na área.

Os elevados valores de CV verificados para alguns atributos sugerem alta heterogeneidade em torno da média entre os atributos químicos nos compartimentos do terreno. Referida heterogeneidade pode ter várias causas, dentre as quais merecem destaque: processos de formação do solo, acúmulo e distribuição das partículas do solo em função da forma do relevo (convexo, retilíneo e côncavo) e do fluxo de água na área. Outras causas que devem ser consideradas se associam ao fato do solo não ter sido cultivado (área com vegetação nativa), favorecendo a formação de gradientes de nutrientes na área.

Em áreas com vegetação nativa há maior integração entre a cobertura vegetal e os atributos do solo, uma vez que ocorre decomposição contínua do material orgânico, associada às menores perdas de nutrientes. Desta maneira e se considerando os processos de formação do solo que atuam ao longo do tempo, observa-se variabilidade dos atributos químicos do solo em detrimento do acúmulo preferencial de serrapilheira em determinadas superfícies, seguido pela decomposição e liberação de nutrientes (Carrera et al., 2009). O acúmulo

Tabela 1. Estatística descritiva dos atributos químicos do solo em função do compartimento na profundidade de $0-20 \mathrm{~cm}$

\begin{tabular}{|c|c|c|c|c|c|c|c|c|}
\hline Atributos & Compartimento & Média & Mediana & Variação & Assimetria & Curtose & d & CV (\%) \\
\hline \multirow{3}{*}{$\mathrm{pH}\left(\mathrm{H}_{2} \mathrm{O}\right)$} & Convexa & 7,6 & 7,4 & 0,31 & 0,08 & $-1,98$ & 0,002 & 7,4 \\
\hline & Retilínea & 7,7 & 7,9 & 0,20 & $-0,27$ & $-1,72$ & 0,045 & 5,8 \\
\hline & Côncava & 7,3 & 7,2 & 0,20 & 1,11 & $-0,22$ & 0,001 & 6,1 \\
\hline \multirow{3}{*}{$\mathrm{K}^{+}\left(\mathrm{cmol}_{\mathrm{c}} \mathrm{kg}^{-1}\right)$} & Convexa & 1,6 & 1,5 & 0,15 & 0,19 & $-0,11$ & $0,941 \mathrm{~N}$ & 24,5 \\
\hline & Retilínea & 1,6 & 1,7 & 0,26 & $-0,76$ & $-0,15$ & $0,397 \mathrm{~N}$ & 31,8 \\
\hline & Côncava & 1,4 & 1,4 & 0,31 & 0,53 & 0,65 & $0,395 \mathrm{~N}$ & 38,7 \\
\hline \multirow{3}{*}{$\mathrm{Ca}^{2+}\left(\mathrm{cmol}_{\mathrm{c}} \mathrm{kg}^{-1}\right)$} & Convexa & 13,3 & 12,5 & 16,85 & 0,92 & 0,37 & $0,492 \mathrm{~N}$ & 30,9 \\
\hline & Retilínea & 15,8 & 15,9 & 24,38 & $-0,29$ & $-0,96$ & $0,637 \mathrm{~N}$ & 31,2 \\
\hline & Côncava & 16,9 & 16,2 & 20,49 & 0,43 & $-0,88$ & $0,331 \mathrm{~N}$ & 26,8 \\
\hline \multirow{3}{*}{$\mathrm{Mg}^{2+}\left(\mathrm{cmol}_{\mathrm{c}} \mathrm{kg}^{-1}\right)$} & Convexa & 4,6 & 4,9 & 1,76 & $-0,49$ & $-0,61$ & $0,440 \mathrm{~N}$ & 29,0 \\
\hline & Retilínea & 4,4 & 4,8 & 2,04 & $-0,10$ & $-1,69$ & $0,063 \mathrm{~N}$ & 32,5 \\
\hline & Côncava & 5,2 & 4,6 & 2,98 & 0,28 & $-0,86$ & $0,343 \mathrm{~N}$ & 33,0 \\
\hline \multirow{3}{*}{$\mathrm{Na}^{+}\left(\mathrm{cmol}_{\mathrm{c}} \mathrm{kg}^{-1}\right)$} & Convexa & 0,06 & 0,03 & 0,005 & 1,72 & 2,91 & 0,001 & 131,9 \\
\hline & Retilínea & 0,06 & 0,05 & 0,004 & 1,12 & 0,39 & 0,009 & 113,0 \\
\hline & Côncava & 0,08 & 0,05 & 0,007 & 0,86 & $-0,69$ & 0,005 & 107,1 \\
\hline \multirow{3}{*}{$\mathrm{H}+\mathrm{Al}\left(\mathrm{cmol}_{\mathrm{c}} \mathrm{kg}^{-1}\right)$} & Convexa & 1,1 & 1,1 & 0,80 & 0,27 & $-1,41$ & $0,054 \mathrm{~N}$ & 84,8 \\
\hline & Retilínea & 0,9 & 0,6 & 0,79 & 0,48 & $-1,54$ & 0,012 & 103,4 \\
\hline & Côncava & 1,4 & 1,8 & 0,91 & $-0,56$ & $-1,14$ & 0,012 & 66,2 \\
\hline \multirow{3}{*}{ CTC $\left(\mathrm{cmol}_{\mathrm{C}} \mathrm{kg}^{-1}\right)$} & Convexa & 20,5 & 21,1 & 13,76 & 0,41 & $-0,59$ & $0,302 \mathrm{~N}$ & 18,1 \\
\hline & Retilínea & 22,7 & 22,6 & 24,62 & $-0,99$ & 2,15 & $0,309 \mathrm{~N}$ & 21,8 \\
\hline & Côncava & 25,1 & 23,5 & 23,70 & 0,72 & $-0,78$ & 0,020 & 19,4 \\
\hline \multirow{3}{*}{ Corg $\left(\mathrm{g} \mathrm{kg}^{-1}\right)$} & Convexa & 17,3 & 15,5 & 34,65 & 1,83 & 4,66 & 0,004 & 34,1 \\
\hline & Retilínea & 16,2 & 14,5 & 47,70 & 1,76 & 4,09 & 0,013 & 42,6 \\
\hline & Côncava & 17,0 & 18,0 & 22,24 & $-0,26$ & $-0,96$ & 0,368 & 27,7 \\
\hline
\end{tabular}

$\mathrm{N}=$ Distribuição normal para $\mathrm{p}>0,05$ - Teste de Shapiro-Wilk 
Tabela 2. Estatística descritiva dos atributos químicos do solo em função da forma do compartimento na profundidade de $20-40 \mathrm{~cm}$

\begin{tabular}{|c|c|c|c|c|c|c|c|c|}
\hline Atributos & Compartimento & Média & Mediana & Variação & Assimetria & Curtose & d & CV (\%) \\
\hline \multirow{2}{*}{$\mathrm{pH}\left(\mathrm{H}_{2} \mathrm{O}\right)$} & Convexa & 7,4 & 7,2 & 0,37 & 0,67 & $-1,29$ & 0,013 & 8,8 \\
\hline & Côncava & 7,2 & 7,1 & 0,21 & 1,05 & 0,51 & 0,045 & 6,3 \\
\hline \multirow[t]{3}{*}{$\mathrm{K}^{+}\left(\mathrm{cmol}_{\mathrm{c}} \mathrm{kg}^{-1}\right)$} & Retilínea & 0,9 & 0,9 & 0,12 & 0,07 & $-0,30$ & $0,879 \mathrm{~N}$ & 38,8 \\
\hline & Côncava & 0,8 & 0,7 & 0,08 & 0,47 & $-1,16$ & $0,066 \mathrm{~N}$ & 35,4 \\
\hline & Convexa & 12,0 & 11,4 & 13,00 & $-0,06$ & $-1,36$ & $0,351 \mathrm{~N}$ & 30,0 \\
\hline $\mathrm{Ca}^{2+}\left(\mathrm{cmol}_{\mathrm{c}} \mathrm{kg}^{-1}\right)$ & Convexa & 5,0 & 4,5 & 2,04 & 2,26 & 4,74 & 0,0002 & 28,5 \\
\hline \multirow[t]{3}{*}{$\mathrm{Mg}^{2+}\left(\mathrm{cmol}_{\mathrm{c}} \mathrm{kg}^{-1}\right)$} & Retilínea & 4,2 & 3,6 & 2,49 & 2,00 & 2,78 & 0,0001 & 37,4 \\
\hline & Côncava & 5,5 & 5,7 & 3,90 & $-0,23$ & $-0,87$ & $0,681 \mathrm{~N}$ & 36,0 \\
\hline & Convexa & 0,08 & 0,06 & 0,004 & 1,32 & 1,49 & 0,047 & 89,8 \\
\hline \multirow[t]{2}{*}{$\mathrm{Na}^{+}\left(\mathrm{cmol}_{\mathrm{c}} \mathrm{kg}^{-1}\right)$} & Retilínea & 0,1 & 0,10 & 0,006 & 0,38 & $-0,79$ & $0,703 \mathrm{~N}$ & 71,1 \\
\hline & Côncava & 0,2 & 0,09 & 0,03 & 1,48 & 2,07 & 0,006 & 99,2 \\
\hline \multirow[t]{3}{*}{ CTC $\left(\mathrm{cmol}_{\mathrm{c}} \mathrm{kg}^{-1}\right)$} & Retilínea & 21,9 & 22,6 & 27,75 & $-1,23$ & 2,43 & $0,135 \mathrm{~N}$ & 24,0 \\
\hline & Côncava & 24,0 & 22,7 & 24,11 & 0,81 & $-0,01$ & $0,089 \mathrm{~N}$ & 20,4 \\
\hline & Convexa & 8,2 & 8,4 & 1,19 & 0,19 & $-1,01$ & $0,584 \mathrm{~N}$ & 13,3 \\
\hline \multirow{2}{*}{ Corg $\left(\mathrm{g} \mathrm{kg}^{-1}\right)$} & Retilínea & 6,8 & 7,2 & 4,16 & $-0,01$ & $-0,76$ & $0,848 \mathrm{~N}$ & 30,1 \\
\hline & Côncava & 7,1 & 7,2 & 2,08 & $-1,51$ & 3,37 & 0,024 & 20,4 \\
\hline
\end{tabular}

$\mathrm{N}=$ Distribuição normal para $\mathrm{p}>0,05$ - Teste de Shapiro-Wilk

da serrapilheira em locais mais baixos geralmente promove maior variabilidade em razão da maior ciclagem de nutrientes e atividade microbiana.

Os atributos químicos do solo diferiram nos compartimentos do terreno, como pode ser observado nas Tabelas 1 e 2; as maiores médias dos atributos nas duas profundidades do solo ocorreram no compartimento côncavo (Tabelas 1 e 2).

Nizeyimana \& Bicki (1992) observaram maiores valores de CTC em terrenos côncavos, atribuindo-os às condições de drenagem da área e às características convergentes da inclinação do relevo.

A superfície côncava apresentou menores valores de $\mathrm{pH}$, porém na área não foram observados problemas de acidez do solo ou alcalinidade excessiva. Esta diminuição dos valores de $\mathrm{pH}$ do solo nessa superfície pode influenciar na disponibilidade de nutrientes, principalmente micronutrientes. Portanto, antes do preparo do solo e da adubação deve-se verificar a disponibilidade de micronutrientes. Na profundidade de 0-20 $\mathrm{cm}$ os valores de $\mathrm{pH}$ variaram de 7,3 a 7,7 (Tabela 1) caracterizando solos com alcalinidade de baixa a média, segundo as recomendações de calagem para o Estado do Ceará (UFC, 1993). A reação alcalina do solo se deve à presença de carbonatos provenientes do material de origem (solos originados de calcário da Formação Jandaíra); nas profundidades de 0-20 e 20-40 cm, foram verificadas tendência de diminuição do valor de $\mathrm{pH}$ no compartimento côncavo e maior variabilidade espacial no retilíneo quando comparadas com as superfícies convexa e côncava (Figura 2).

A acidez potencial $(\mathrm{H}+\mathrm{Al})$ foi predominantemente formada pelos íons $\mathrm{H}^{+}$, uma vez que os teores de $\mathrm{Al}^{3+}$ não foram expressivos. Canellas et al. (2003) observaram maiores valores de acidez potencial na camada superficial nas áreas com menor teor de carbono indicando que a oxidação da matéria orgânica pode ser um componente importante na geração da acidez. Os maiores valores de acidez potencial foram verificados na camada de 20-40 cm no microrrelevo côncavo (Figura 4). Os valores encontrados para essa característica foram baixos, concordando com as variações de $\mathrm{pH}$ observadas na área.

Solos de origem calcária têm, como característica, valores elevados de $\mathrm{pH}$ e alto teor de cálcio, o que interfere no processo de adsorção e solubilização de íons, responsáveis pelo teor de nutrientes no solo. $\mathrm{O}$ pH mais elevado do solo favorece a troca

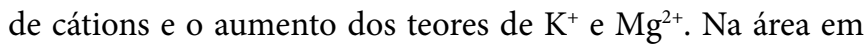
estudo pode-se constatar que, mesmo não recebendo qualquer adubação ou correção de acidez, os valores de cátions trocáveis foram elevados (Tabelas 1 e 2).

Os teores médios de $\mathrm{K}^{+}$não variaram nas superfícies retilínea e convexa enquanto que teores de carbono orgânico (Corg) foram superiores na superfície convexa (Tabelas $1 \mathrm{e}$ 2). Estudos desenvolvidos em Latossolos demonstraram que áreas com microrrelevo côncavo e convexo, independentes do uso e manejo, apresentaram maior variabilidade dos atributos químicos do que áreas de relevo retilíneo (Souza et al., 2006). Na medida em que as áreas passam a ser cultivadas, essas diferenças podem interferir no manejo da adubação.

Os teores mais elevados de $\mathrm{K}^{+}$foram constatados na profundidade de $0-20 \mathrm{~cm}$ (Tabela 1); porém, não foi detectada dependência espacial. Na profundidade de $20-40 \mathrm{~cm}$ foi constatado elevado teor de $\mathrm{K}^{+}$na superfície convexa, o que pode ser explicado pelo intemperismo dos materiais primários do solo. Em cada superfície ocorreu variação no teor de $\mathrm{K}^{+}$(Figura 
A. $\mathrm{pH} 0-20 \mathrm{~cm}$

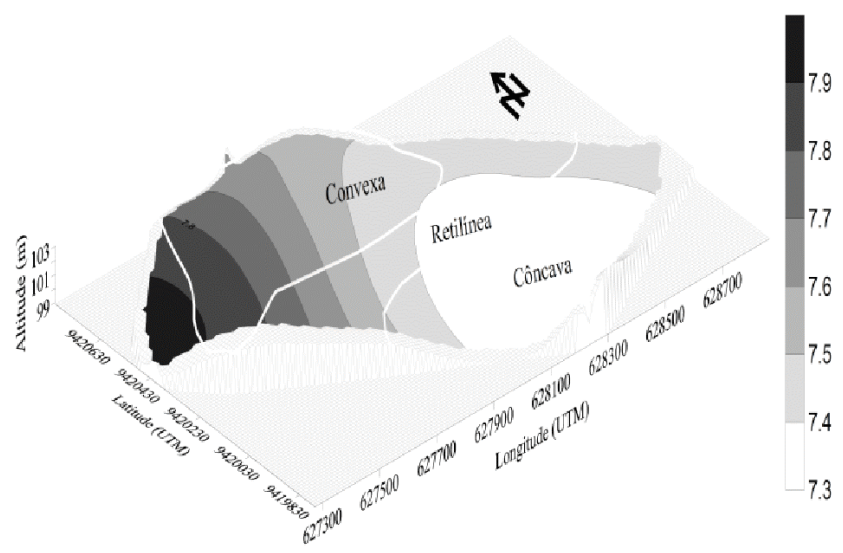

C. $\mathrm{Ca}^{2+}\left(\mathrm{cmol}_{\mathrm{c}} \mathrm{kg}^{-1}\right) 0-20 \mathrm{~cm}$

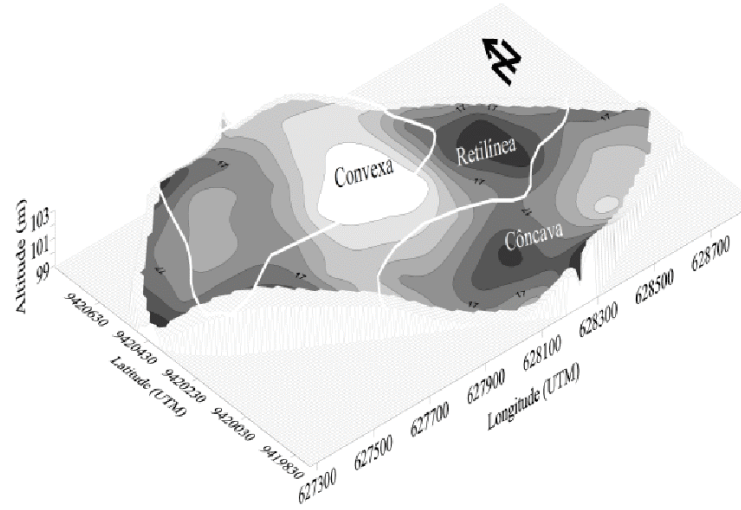

E. $\mathrm{Mg}^{2+}\left(\mathrm{cmol}_{\mathrm{c}} \mathrm{kg}^{-1}\right) 0-20 \mathrm{~cm}$

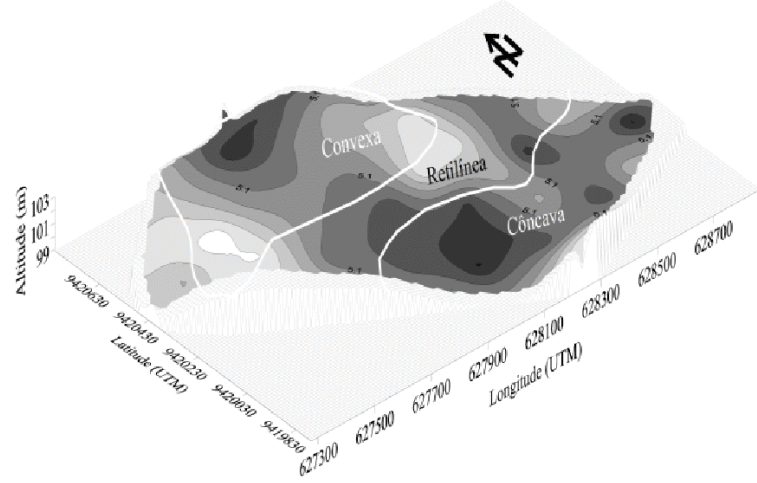

B. $\mathrm{pH} 20-40 \mathrm{~cm}$

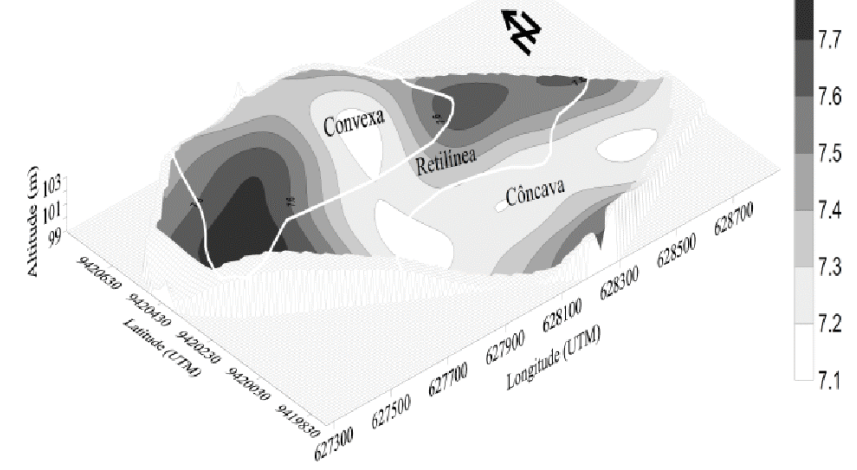

D. $\mathrm{Ca}^{2+}\left(\mathrm{cmolc} \mathrm{kg}^{-1}\right) 20-40 \mathrm{~cm}$

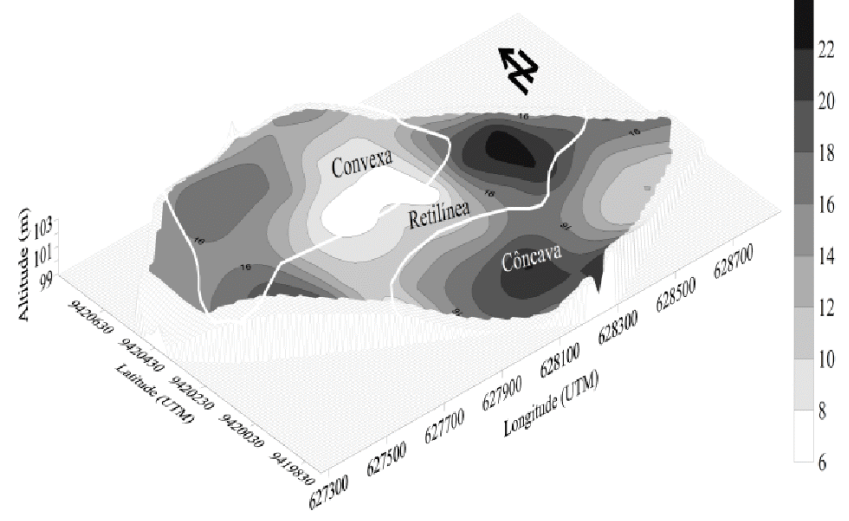

F. $\mathrm{Mg}^{2+}\left(\mathrm{cmol}_{\mathrm{c}} \mathrm{kg}^{-1}\right) 20-40 \mathrm{~cm}$

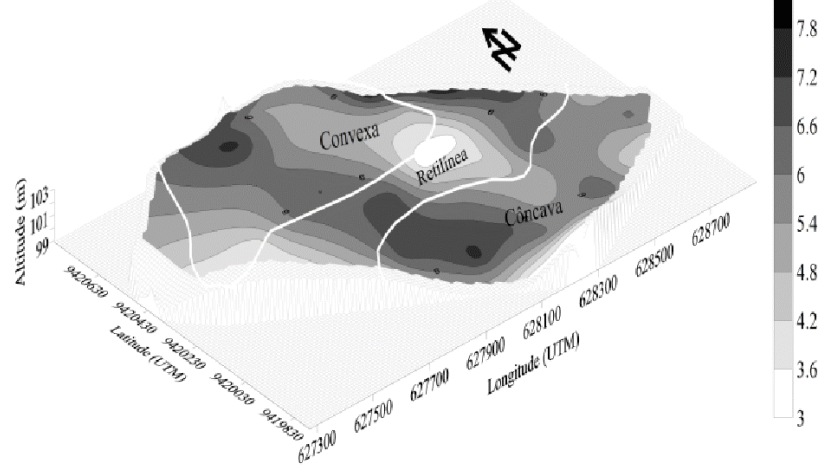

Figura 2. Distribuição espacial do $\mathrm{pH}, \mathrm{Ca}^{2+}\left(\mathrm{cmol}_{\mathrm{c}} \mathrm{kg}^{-1}\right)$ e $\mathrm{Mg}^{2+}\left(\mathrm{cmol}_{\mathrm{c}} \mathrm{kg}^{-1}\right)$ nas profundidades de 0-20 e 20-40 cm, em função da forma do relevo

3) haja vista que este nutriente apresenta alta mobilidade que facilita seu transporte com o fluxo de água acarretando em características químicas diferenciadas na área (Vidal-Torrado et al., 2005).

Os teores de $\mathrm{Ca}^{2+}$ e $\mathrm{Mg}^{2+}$ foram elevados, conforme classificação de Tomé Júnior (1997). Esses teores elevados advêm da origem calcária do solo. Pela Figura 2 observa-se que os valores de $\mathrm{Ca}^{2+}$ e de $\mathrm{Mg}^{2+}$ apresentaram pouca variação entre as profundidades estudadas, além de que foram observados teores de $\mathrm{Ca}^{2+}$ maiores que os de $\mathrm{Mg}^{2+}$, o que é comum em solos bem drenados e que não receberam calagem.

As relações Ca:Mg observadas para os solos nas superfícies côncava, retilínea e convexa, foram 2,9:1; 3,6:1 e 2,4:1, respectivamente. Considerando o interesse em estabelecer um bananal na área de estudo, a superfície convexa apresenta relação Ca:Mg mais adequada, pois se enquadra na faixa de 1,5:1 a 3:1 indicada por Silva et al. (1999) como ideal para bananeiras. Já as superfícies côncava e retilínea precisam de 
A. $\mathrm{K}^{+}\left(\mathrm{cmol}_{\mathrm{c}} \mathrm{kg}^{-1}\right) 20-40 \mathrm{~cm}$

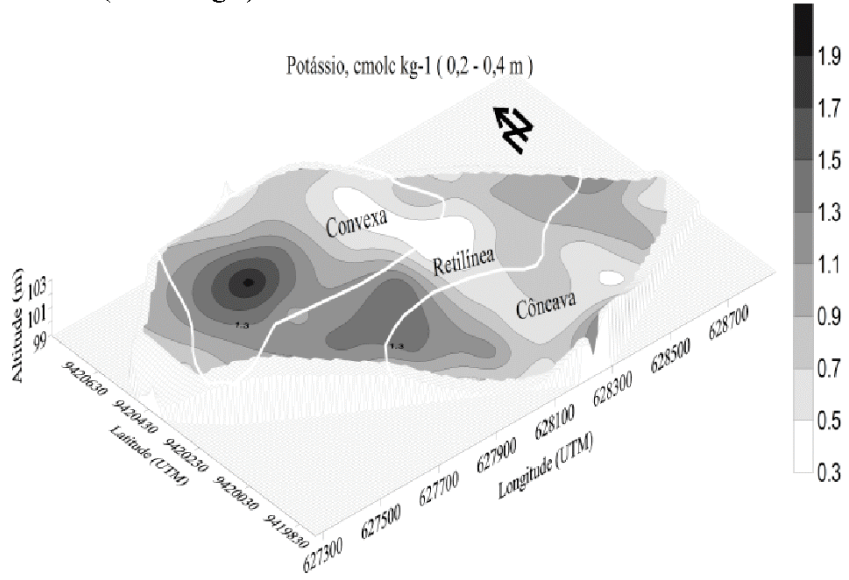

B. Corg $\left(\mathrm{g} \mathrm{kg}^{-1}\right) 0-20 \mathrm{~cm}$

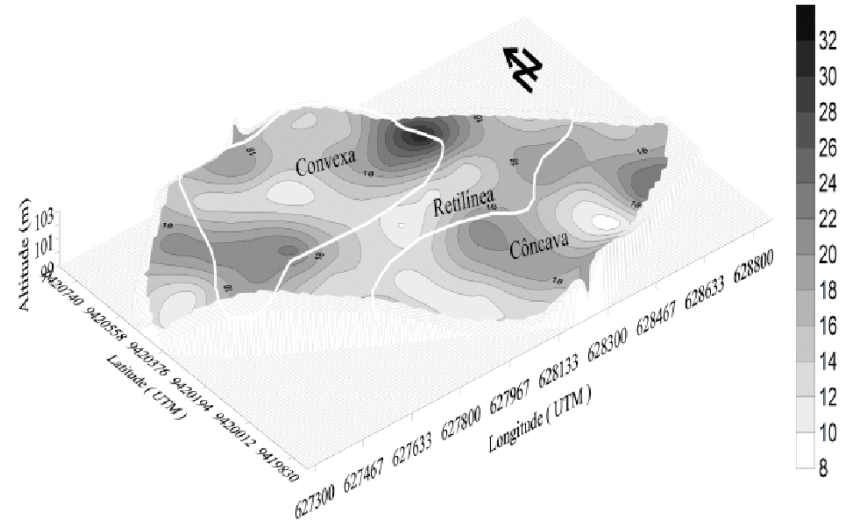

D. $\mathrm{Na}^{+}\left(\mathrm{cmol}_{\mathrm{c}} \mathrm{kg}^{-1}\right) 0-20 \mathrm{~cm}$

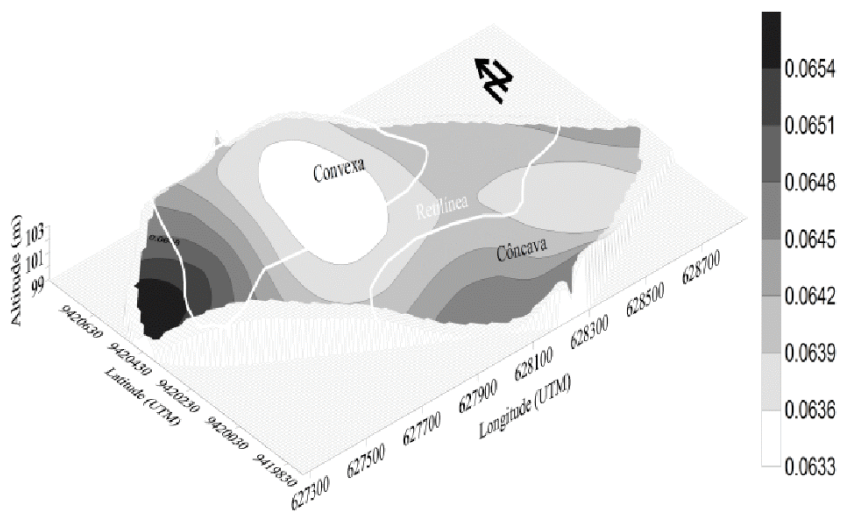

C. Corg $\left(\mathrm{g} \mathrm{kg}^{-1}\right) 20-40 \mathrm{~cm}$

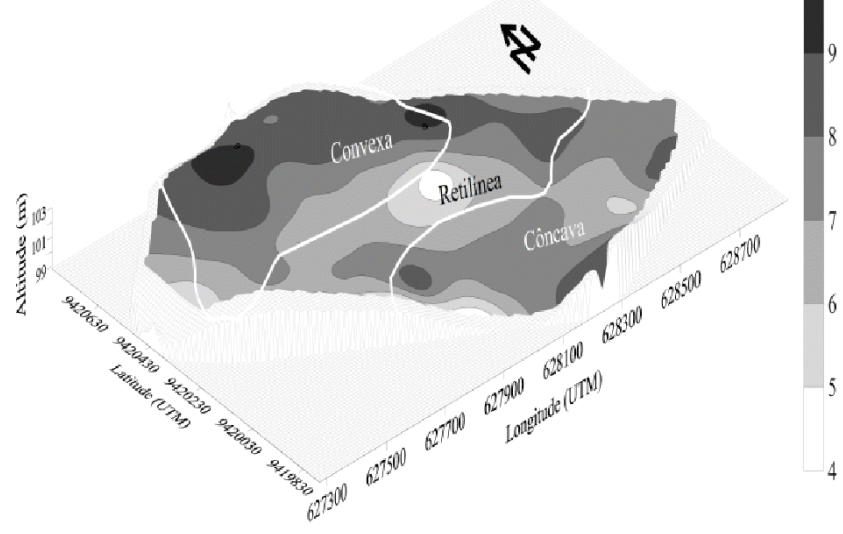

E. $\mathrm{Na}^{+}\left(\mathrm{cmol}_{\mathrm{c}} \mathrm{kg}^{-1}\right) 20-40 \mathrm{~cm}$

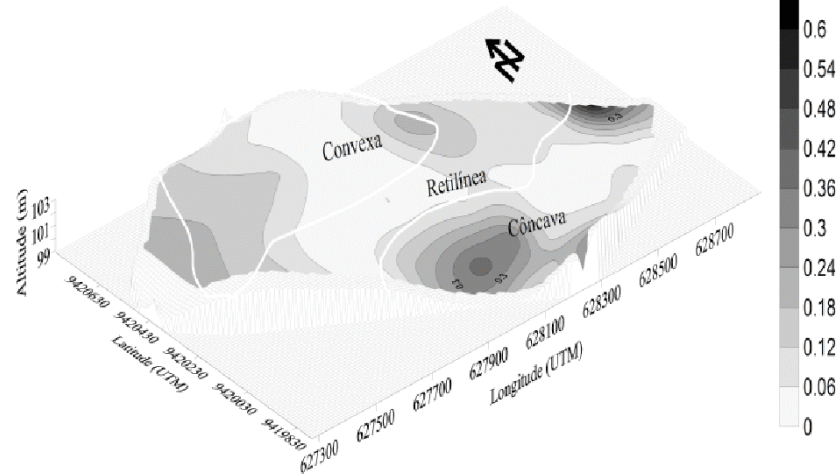

Figura 3. Distribuição espacial do $\mathrm{K}^{+}\left(\mathrm{cmol}_{\mathrm{c}} \mathrm{kg}^{-1}\right)$, Corg $\left(\mathrm{g} \mathrm{kg}^{-1}\right)$ e Na${ }^{+}\left(\mathrm{cmol}_{\mathrm{c}} \mathrm{kg}^{-1}\right)$ nas profundidades de 0-20 e 20-40 $\mathrm{cm}$, em função da forma do relevo

atenção, sendo pertinente recomendar fertilizante que contenha Mg para aumentar a disponibilidade do nutriente em relação ao Ca. A explicação para os maiores teores de nutrientes na camada superficial do solo está associada à vegetação nativa a qual, por sua vez, está associada à ciclagem mais eficiente de nutrientes justificando as tendências do $\mathrm{Ca}^{2+}, \mathrm{Mg}^{2+}$ e também os teores mais elevados de $\mathrm{K}^{+}$na camada superficial. Silva et al. (2007) observaram, trabalhando com coberturas vegetais em
Chernossolo, maiores valores $\mathrm{K}^{+}$e $\mathrm{Ca}^{2+}$ na camada superficial para o tratamento caracterizado pela presença da mata.

A CTC foi elevada em toda a área avaliada (Tabela 1) obtendo-se valores médios de 22,81 e 21,50 $\mathrm{cmol}_{\mathrm{c}} \mathrm{kg}^{-1}$ para as profundidades de 0-20 e 20-40 cm, respectivamente. Os teores de $\mathrm{Al}^{+3}$ trocável foram muito baixos, o que está associado tanto ao fato dos solos serem de clima semiárido, em que os teores deste cátion não são expressivos, quanto devido aos valores de 
pH do solo estarem acima de 5,5 e, principalmente, neste estudo com solo de origem calcária.

Maiores valores de CTC normalmente ocorrem em locais onde os teores de $\mathrm{MO}$ e argila são elevados. Esses atributos contribuem com o aumento de cargas negativas favorecendo, assim, a retenção de nutrientes. Outro fator que deve ser considerado na área estudada é a inclinação no terreno da superfície convexa até a côncava o que, provavelmente, contribui com o acúmulo de argila e incremento da CTC nessa superfície. Para Rawls \& Pachepsky (2002), o microrrelevo côncavo contribui para a remoção de componentes orgânicos e minerais das áreas mais inclinadas e com a deposição em posições do relevo consideradas mais estáveis, menos inclinadas.

Os elevados valores de CTC na área de estudo estão associados aos minerais da fração argila de modo que, embora haja predominância de caulinita, alguns autores mostram em seus trabalhos a ocorrência de ilita em solos da Chapada do Apodi, estimando-se, com base em análise semiquantitativa, a proporção de $10 \%$ desse argilomineral (Mota et al., 2007).

Quanto aos teores de Corg, no microrrelevo convexo na camada de 0-20 cm ocorreu variação de 8 a $32 \mathrm{~g} \mathrm{~kg}^{-1}$, indicando grande variabilidade dentro de cada superfície (Figura 3 ).

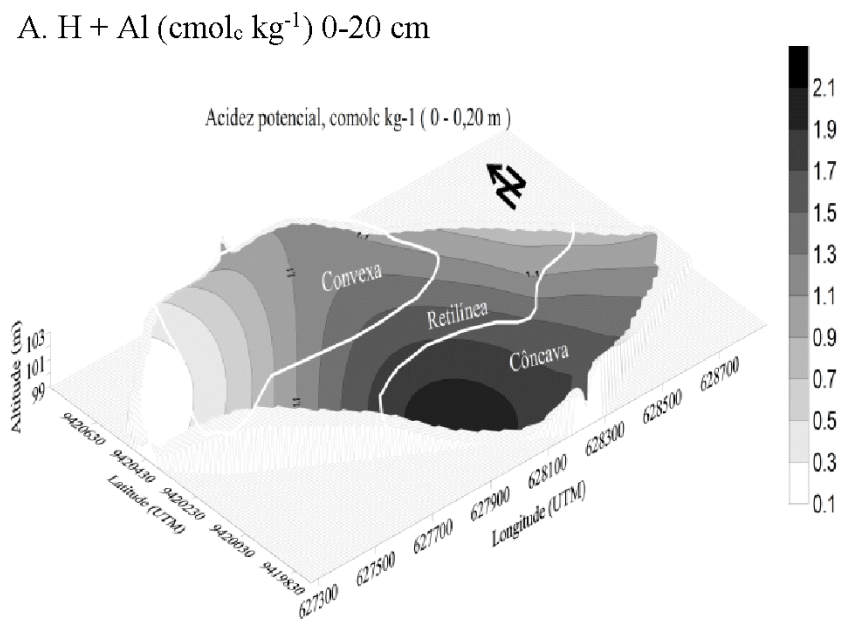

C. CTC $\left(\mathrm{cmol}_{\mathrm{c}} \mathrm{kg}^{-1}\right)$ 0-20 cm

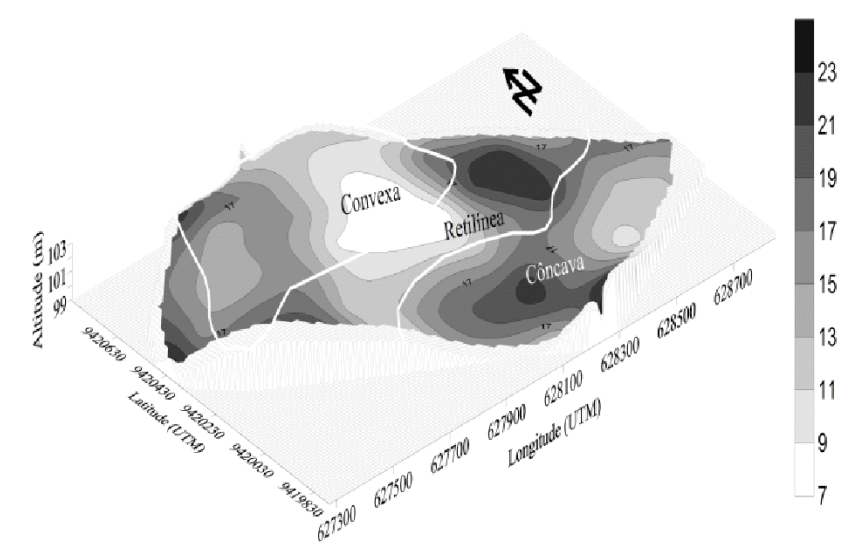

Os teores de $\mathrm{Na}^{+}$foram superiores na camada de $20-40 \mathrm{~cm}$ e no microrrelevo côncavo (Tabela 2), o que se justifica pelo fluxo de água que favoreceu a movimentação horizontal e vertical desse nutriente, bem como seu acúmulo nas camadas mais profundas. As formas do relevo influenciam no tempo de exposição do material, na intensidade e no fluxo da água no perfil do solo (Campos et al., 2006).

Os modelos ajustados pelo semivariograma nas camadas de 0-20 e 20-40 cm apresentaram dependência espacial (Tabela 3), ou seja, a distribuição no espaço desses atributos não é aleatória, com exceção do $\mathrm{K}^{+}$na camada de $0-20 \mathrm{~cm}$.

Para a maioria dos atributos químicos nas duas profundidades em questão foi observada forte dependência espacial (Tabela 3), atribuída a fatores intrínsecos, tais como: material de origem, relevo, clima, organismos e tempo (Cambardella et al., 1994). A dependência espacial não é atribuída a fatores extrínsecos visto que na área não houve influência de cultivo, adubação, calagem, preparo do solo nem de tráfego de maquinaria agrícola, dentre outros. Desta forma, tem-se a confirmação da influência do microrrelevo e do fluxo de água na variabilidade espacial dos atributos químicos do solo.

B. $\mathrm{H}+\mathrm{Al}\left(\mathrm{cmol}_{\mathrm{c}} \mathrm{kg}^{-1}\right) 20-40 \mathrm{~cm}$

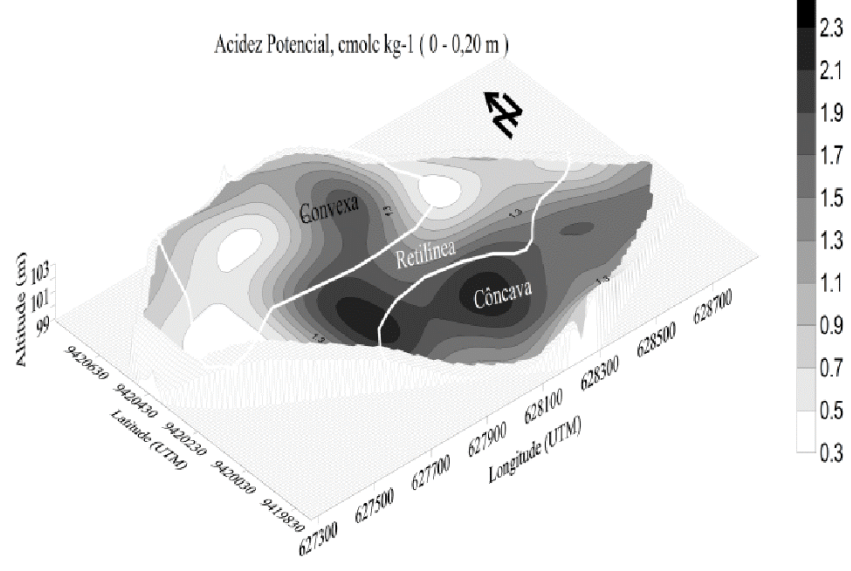

D. CTC ( $\left.\mathrm{cmol}_{\mathrm{c}} \mathrm{kg}^{-1}\right) 20-40 \mathrm{~cm}$

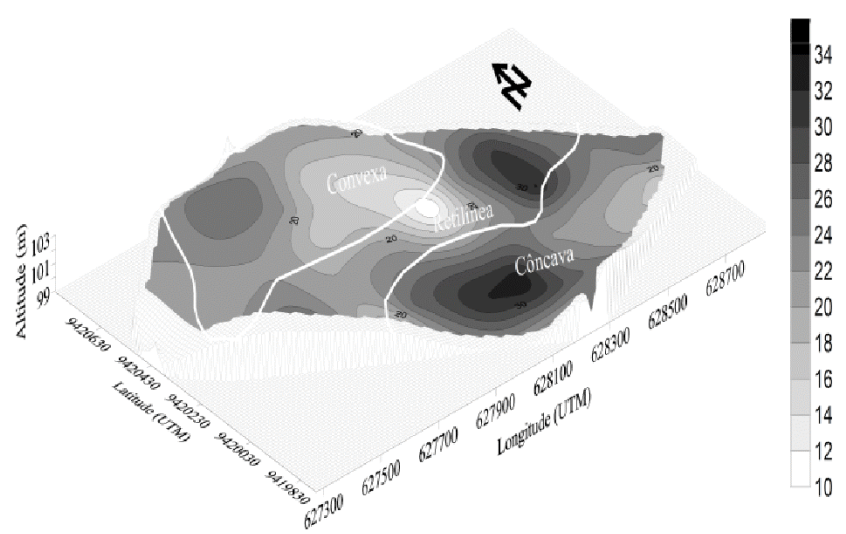

Figura 4. Distribuição espacial do $\mathrm{H}+\mathrm{Al}\left(\mathrm{cmol}_{\mathrm{c}} \mathrm{kg}^{-1}\right)$ e $\mathrm{CTC}\left(\mathrm{cmol}_{\mathrm{c}} \mathrm{kg}^{-1}\right)$ nas profundidades de 0-20 e 20-40 $\mathrm{cm}$, em função da forma do relevo 
Tabela 3. Modelos e parâmetros estimados dos semivariogramas para os atributos químicos do solo nas profundidades de $0-20$ e 20-40 cm, independente do microrrelevo

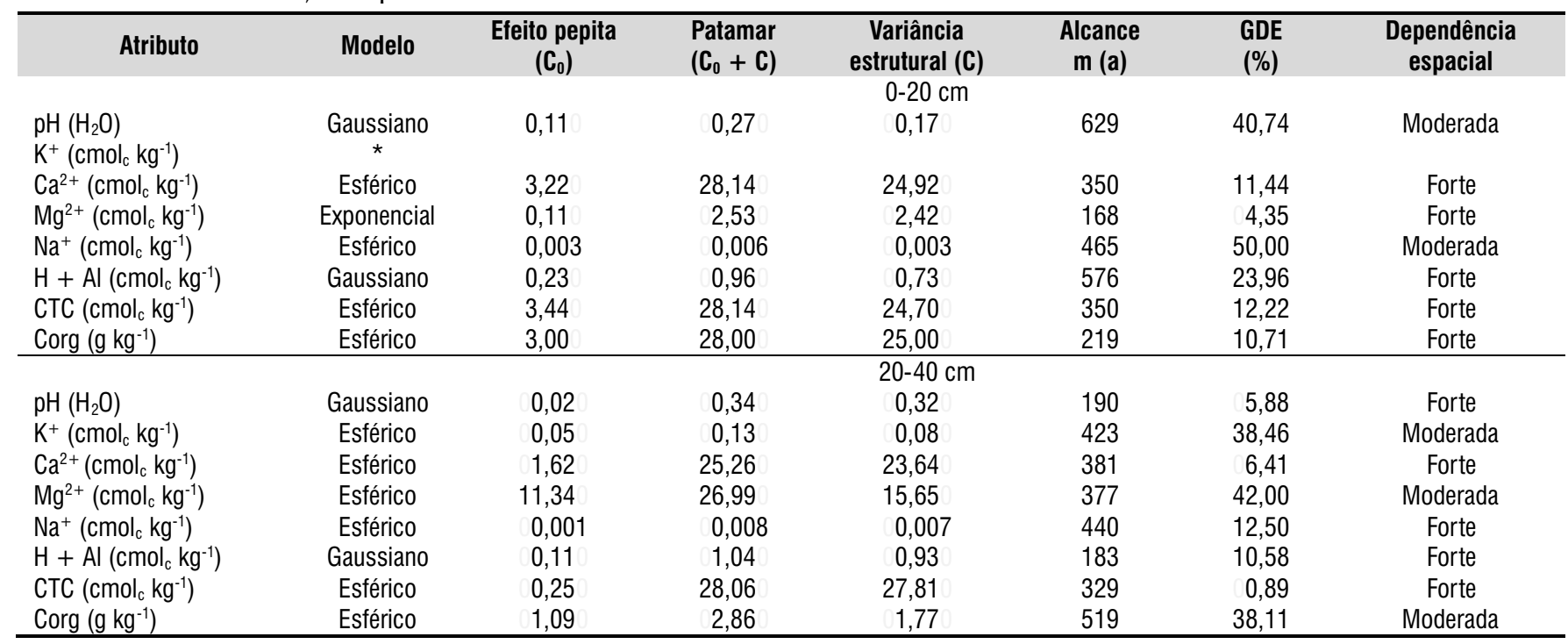

Grau de dependência espacial $(\mathrm{GDE})=\left[\mathrm{C}_{0} /\left(\mathrm{C}_{0}+\mathrm{C}\right)\right] \times 100$

Os modelos esférico e gaussiano foram os que melhor se ajustaram aos semiviariogramas dos atributos químicos do solo nas duas profundidades avaliadas, com exceção do $\mathrm{Mg}^{2+}$ na profundidade de $0-20 \mathrm{~cm}$ que se ajustou ao modelo exponencial (Tabela 3). No ajuste esférico todos os pontos pertencentes a um círculo com o raio igual ao alcance (distância a partir da qual as amostras não se correlacionam) são similares a ponto de poder ser utilizados para estimar valores para qualquer ponto entre eles (Machado et al., 2007).

O conhecimento do alcance da dependência espacial permite que seja definido o raio de amostragem de maneira a garantir, nas próximas amostragens e para as mesmas condições deste estudo, a independência dos pontos amostrais, a minimização do erro padrão da média e também uma maneira de diminuir o número de amostras a serem coletadas.

Os atributos químicos do solo apresentaram valores de alcance elevados indicando eficácia da grade de amostragem na detecção da variação espacial desses atributos. Por meio dos valores de alcance também foi possível observar a variação espacial existente entre os atributos químicos em cada profundidade visto que os valores foram variados (Tabela 3).

\section{Conclusões}

1. Os atributos químicos do solo variaram espacialmente.

2. O microrrelevo influencia na direção do fluxo de água e induz à variabilidade espacial dos atributos químicos do solo.

3. O microrrelevo determina áreas com características diferenciadas em relação ao $\mathrm{pH}$ e à disponibilidade de nutrientes devendo ser considerado na definição de áreas mais homogêneas e na utilização de técnicas de manejo diferenciadas para a implantação de culturas.

\section{Literatura Citada}

Bockheim, J. C.; Gennadiyev, A. N.; Hammer, R. D.; Tandarich, J. P. Historical development of key concepts in pedology. Geoderma, v.124, p.23-26, 2005.

Brasil. Ministério da Agricultura. Levantamento exploratório e reconhecimento de solos do Estado do Ceará. Recife: EMBRAPA, v.1. 1973. 297p.

Cambardella, C. A.; Moorman, T. B.; Novak, J. M.; Parkin, T. B.; Karlen, D. L.; Turco, R. F.; Konopka, A. E. Field-scale variability of soil properties in Central Iowa Soils. Soil Science Society of American Journal, v.58, p.1501-1511, 1994.

Campos, M. C. C.; Cardozo, N. P.; Marques Júnior, J. Modelos de paisagem e sua utilização em levantamentos pedológicos. Revista de Biologia e Ciências da Terra, v.6, p.104-114, 2006.

Canellas, L. P.; Velloso, A. C. X.; Marciano, C. R.; J. F. G. P. Ramalho, J. F. G. P.; Rumjanek. V. M.; Rezende, C. E.; Santos, G. A. Chemical soil properties of an inceptisol under longterm sugarcane crops with vinasse application and without slash burning. Revista Brasileira de Ciência do Solo, v.27, p.935-944, 2003.

Carrera, A. L.; Mazzarino, M. J.; Bertiller. M. B.; Del Valle, H. F.; Carretero, E. M. Plant impacts on nitrogen and carbon cycling in the Monte Phytogeographical Province, Argentina. Journal of Arid Environments, v.73, p.192-201, 2009.

Claessen, M. E. C. (org.). Manual de métodos de análise de solo. 2.ed. Rio de Janeiro: Embrapa CNPS, 1997. 212p. Documentos, 1

Corá, J. E.; Araújo, A. V.; Pereira, G. T.; Beraldo, J. M. G. Variabilidade espacial de atributos do solo para adoção do sistema de agricultura de precisão na cultura de cana de açúcar. Revista Brasileira de Ciência do Solo, v.28, p.10131021, 2004. 
Cruz, J. S.; Assis Júnior, R. N.; Matias, R. S. S.; CamachoTamayo, J. H.; Tavares, R. C. Análise espacial de atributos físicos e carbono orgânico em Argissolo Vermelho-Amarelo cultivado com cana de açúcar. Ciência e Agrotecnologia, v.34, p.271-278, 2010.

Daniels, R. B.; Hammer, R. D. Soil geomorphology. New York: Jonh Willey, 1992. 236p.

Franzen, D. W.; Nanna, T.; Norvell, W. A. A survey of soil attributes in North Dakota by landscape position. Agronomy Journal, v.98, p.1015-1022, 2006.

Gobin, A.; Campling, P.; Feyen, J. Soil-landscape modelling to quantify spatial variability of soil texture. Physics and Chemistry of the Earth, Part B: Hydrology, Oceans and Atmosphere, v.26, p.41-45, 2001.

Golden Software Inc. Surfer for windows: realese 7.0, contouring and 3D surface mapping for scientist's engineers user's guide. New York: New York Golden, 1999. 619p.

Guo, L. B.; Wang, M. B.; Gifford, R. The change of soil carbon stocks and fine root dynamics after land use change from a native pasture to a pine plantation. Plant and Soil, v.299, p.251-262, 2007.

Machado, L. D.; Lana, A. M. Q.; Lana, R. M. Q.; Guimarães, E. C.; Ferreira, C. V. Variabilidade espacial de atributos químicos do solo em áreas sob sistema de plantio convencional. Revista Brasileira de Ciência do Solo, v.31, p.591-599, 2007.

Marques Júnior, J.; Lepsch, I. F. Depósitos superficiais neocenozóicos, superfícies geomórficas e solos em Monte Alto, SP. Geociências, v.19, p.265-281, 2000.

Mello, G.; Bueno, C. R. P.; Pereira, G. T. Variabilidade espacial das propriedades físicas e químicas do solo em áreas intensamente cultivadas. Revista Brasileira de Engenharia Agrícola e Ambiental, v.10, p.294-305, 2006.

Montezano, Z. F.; Corazza, E. J.; Muraoka, T. Variabilidade espacial da fertilidade do solo em área cultivada e manejada homogeneamente. Revista Brasileira de Ciência do Solo, v.30, p.839-847, 2006.

Mota, J. C. A.; Assis Júnior, R. N.; Amaro Filho, J.; Romero, R. E.; Mota, F. O. B.; Libardi, P. L. Atributos mineralógicos de três solos explorados com a cultura do melão na Chapada do Apodi - RN. Revista Brasileira de Ciência do Solo, v.31, p.445-454, 2007.
Nizeyimana, E.; Bicki, T. J. Soil and soil landscape relationships in the North Central region of Rwanda, East-Central Africa. Soil Science, v.153, p.225-236, 1992.

Oliveira, P. C. G.; Farias, P. R. S.; Lima, H. V.; Fernandes, A. R.; Oliveira, F. A.; Pita, J. D. Variabilidade espacial de propriedades químicas do solo e da produtividade de citros na Amazônia Oriental. Revista Brasileira de Engenharia Agrícola e Ambiental, v.13, p.708-715, 2009.

Rawls, W. J.; Pachepsky, Y. A. Using field topographic to estimate soil water retention. Soil Science, v.167, p.423-435, 2002.

Silva, J. T. A.; Borges, A. L.; Malburg, J. L. Solos, adubação e nutrição da bananeira. Informe Agropecuário, v.20, p.2136, 1999.

Silva, R. C.; Pereira, J. M.; Araújo, Q. R.; Pires, A. J. V.; Del Rei, A. J. Alterações nas propriedades químicas e físicas de um Chernossolo com diferentes coberturas vegetais. Revista Brasileira de Ciência do Solo, v.31, p.101-107, 2007.

Souza, Z. M.; Marques Júnior, J.; Pereira, G. T.; Montanari, R. Otimização amostral de atributos de Latossolos considerando aspectos solo-relevo. Ciência Rural, v.36, p.829-836, 2006.

Tomé Júnior, J. B. Manual para interpretação de análise de solo. Guaiba: Livraria e Editora Agropecuária. 1997. 87p.

UFC - Universidade Federal do Ceará. Recomendações de adubação e calagem para o estado do Ceará. Fortaleza: UFC, 1993. 247p.

Vidal-Torrado, P.; Lepsch, I. F.; Castro, S. S. Conceitos e aplicações das relações pedologia-geomorfologia em regiões tropicais úmidas. In: Vidal-Torrado, P.; Alleoni, L. R. F.; Cooper, M.; Silva, A. P.; Cardoso, E. J.; Prochnow, L. I. (org.). Tópicos em Ciência do Solo. Viçosa: SBCS, 2005. v.4, p.145-192.

Warrick, A. W.; Nielsen, D. R. Spatial variability of soil physical properties in the field. In: Hillel, D. (ed). Aplications of soil physics. New York: Academic Press, 1980. 385p.

Zanão Júnior, L. A.; Lana, R. M. Q.; Guimarães, E. C.; Pereira, J. M. A. Variabilidade espacial dos teores de macronutrientes em Latossolos sob sistema plantio direto. Revista Brasileira de Ciência do Solo, v.34, p.389-400, 2010 . 